Check for updates

Cite this: RSC Adv., 2017, 7, 46036

Received 4th May 2017

Accepted 4th September 2017

DOI: $10.1039 / c 7 r a 04999 d$

rsc.li/rsc-advances

\section{Biocompatibility and safety evaluation of a silk fibroin-doped calcium polyphosphate scaffold copolymer in vitro and in vivo}

\author{
Huixu Xie, $\dagger^{\text {abcd }}$ Jianyun Wang, $\dagger^{\text {ef }}$ Yan He, $\dagger^{\text {ad }}$ Zhipeng Gu, ${ }^{9}$ Jia Xu, ${ }^{a}$ Longjiang $\mathrm{Li}^{\star b c}$ \\ and Qingsong Ye*ad
}

For the reconstruction of cartilage and bone defects, bone repair scaffolds with porous network structures have been extensively studied. In our previous study, CPP-type bioceramics showed higher compressive strength and enhanced degradation after silk fibroin doping, and SF/CPP could be considered a suitable bioceramic for bone tissue-engineering. The aim of this study was to evaluate the biocompatibility and safety of SF/CPP in vitro and in vivo. The cell biocompatibility was evaluated with regard to the cytotoxicity of the scaffolds using co-culture and MTT tests in vitro. The in vivo biocompatibility of SF/ CPP was evaluated by implanting the scaffolds in the subcutaneous and intramuscular regions of experimental animals. We established an experimental animal model to prepare critical-sized cranial defects and evaluated the biodegradability and osteoconductivity of the scaffolds in vivo. The results indicated that the SF/CPP scaffold yielded better biocompatibility and safety performance than the CPP scaffold in vitro and in vivo. Immunohistochemistry staining in vivo for OPN and OCN also indicated that SF/CPP has potential to promote the regeneration of critical-sized cranial defects. The SF/CPP scaffold has good biocompatibility and safety for experimental animals and could also serve as a potential effective bioceramic for a range of bone regeneration applications.

\section{Introduction}

For the reconstruction of cartilage and bone defects, bone repair scaffolds with porous network structures have been extensively studied. The porous scaffolds should bind to the bone layer to substitute for bone defects after implantation; it is believed that the scaffold binds to bone and is then resorbed and replaced by bone. ${ }^{1}$ Calcium polyphosphate (CPP), a resorbable ceramic invented 20 years ago, functions as a bone graft scaffold and has been reported to exhibit bio-resorb ability. ${ }^{2,3}$ This ceramic has been widely researched because of its biocompatibility, ${ }^{4}$

aJCU-WMU Joint Research Group of Tissue Engineering, Wenzhou Medical University, Wenzhou, China

${ }^{b}$ West China School of Stomatology, Sichuan University, Chengdu 610041, China

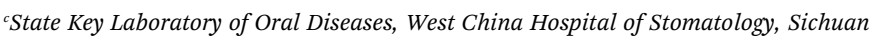
University, Chengdu 610041, China

${ }^{d}$ Regenerative Dentistry, School of Dentistry, University of Queensland, Brisbane, QLD 4006, Australia. E-mail: qingsongye@hotmail.com

${ }^{e}$ Department of Orthopaedics and Traumatology, The University of Hong Kong, 102 Pokfulam Road, Hong Kong SAR, China

${ }^{f}$ Shenzhen Key Laboratory for Innovative Technology in Orthopaedic Trauma, The University of Hong Kong Shenzhen Hospital, 1 Haiyuan First Road, Futian District, Shenzhen 518053, China

${ }^{g}$ Department of Biomedical Engineering, School of Engineering, Sun Yat-sen University, Guangzhou 510006, China

$\dagger$ Contribute equally to this work. controllable degradability, and compositional similarities to natural bone. ${ }^{5}$ However, CPP still shows poor mechanical properties in bone grafting procedures. Furthermore, there is a need for strategies that stimulate bone growth with proper functional properties. Therefore, researchers have tried to develop a few strategies to modify CPP to enhance its mechanical properties.

In our previous studies, we doped strontium into CPP to synthesize strontium-doped calcium polyphosphate (SCPP) with various crystal structures; we also researched the biocompatibility, degradability and mechanical properties of SCPP in vitro ${ }^{6}$ and in vivo. ${ }^{7}$ SCPP is a good bioceramic bone repair scaffold that shows potential to repair bone defects and promote angiogenesis in bone tissue. However, these scaffolds still could not greatly satisfy complex bone repair requirements, such as good mechanical properties and good degradation.

The common naturally derived polymers used to improve mechanical properties consist of two types of bioceramics. One type consists of synthetic biodegradable polymers, such as poly lactic acid (PLA) and poly glycolic acid (PGA). ${ }^{4,8}$ Other naturally derived polymers, such as collagen, chitosan and silk fibroin (SF), are also doped in bioceramics to improve the properties of the scaffolds. ${ }^{9,10}$ Naturally derived polymer silk proteins, which undergo biosynthesis in epithelial cells, are usually produced within specialized glands of silkworms; the proteins are stored prior to spinning into fibers. ${ }^{9}$ Bombyx mori silk protein is the most extensively characterized silkworm silk; it consists of two 
fibroin threads adhered together with sericin gum, resulting in a single thread about 10 to $25 \mu \mathrm{m}$ in diameter. Due to its outstanding properties, including good biocompatibility, water vapor permeability, biodegradability, and minimal inflammatory reactions, the use of SF in biomedical applications has been widely reported recently. ${ }^{\mathbf{9 1 1}}$ In our previous study, silk fibroin was applied to CPP using a simple crosslinking method; we found that the SF/CPP scaffold yielded higher compressive strength and better degradation behavior after Bombyx mori silk was doped into the CPP structure. ${ }^{12}$

In this study, we further studied the SF/CPP porous scaffold; the morphology, structure, mechanical properties, bioactivity and biocompatibility of the composite scaffold were studied in vitro and in vivo.

\section{Experimental section}

\section{Reagents}

Fetal calf serum (FBS), alpha-modified essential medium $(\alpha-$ MEM) and trypsin (in EDTA) were purchased from Gibco (Paisley, UK); other reagents were obtained from Sigma-Aldrich (Poole, UK), except where otherwise stated. Porous SF/CPP, CPP and HA scaffolds for the experiments were prepared according to the methods described in our previous study. ${ }^{13}$

\section{Pre-osteoblast cell culture on scaffolds}

MC3T3E-1 cells were obtained from American Type Culture Collection (ATCC). Cells were cultured in $\alpha$-MEM containing $10 \%$ fetal bovine serum (FBS, Hyclone, Rockford) and 1\% penicillin/streptomycin and were grown at $37{ }^{\circ} \mathrm{C}$ in $5 \% \mathrm{CO}_{2}$. Cells were cultured for $24 \mathrm{~h}$ in a humidified atmosphere of $5 \%$ $\mathrm{CO}_{2}$ at $37^{\circ} \mathrm{C}$ in $25 \mathrm{~cm}^{2}$ flasks until confluent. All scaffold discs were sterilised under UV light for $2 \mathrm{~h}$ per side and were preconditioned in medium; about 20000 MC3T3E-1 cells per $\mathrm{cm}^{2}$ were seeded on the scaffolds and cultured for 1, 7, or 14 days in standard culture medium, which was exchanged three times per week to visualise osteoclast differentiation.

\section{Analysis of cytotoxicity and proliferation of the MC3T3E1 cells by MTT}

$4 \times 10^{3}$ cells per well were seeded in 96-well plates after being cultured overnight. After the cells were cultured for $24 \mathrm{~h}$, the supernatant was discarded; the CPP, HA and SF/CPP scaffolds were sterilised under UV light for $2 \mathrm{~h}$ per side and preconditioned in medium (exchanged every two days). The cell culture medium was added to the MC3T3 E-1 cells on the basis of equal surface area into each well $(n=6)$. The plates were analyzed to evaluate the cell proliferation on the 2nd, 4th and 6th day, respectively. $10 \mu \mathrm{l}$ per well MTT solutions $\left(4 \mathrm{mg} \mathrm{ml}^{-1}\right.$ in concentration) were added to every well after the scheduled time; then, the plate was incubated at $37^{\circ} \mathrm{C}$ for $4 \mathrm{~h}$. Following this, dimethylsulfoxide $(100 \mu \mathrm{l}$ per well) was added to all wells and the plates were shaken for $10 \mathrm{~min}$. The optical density (OD) of each well was measured by a Microplate Reader (Model550, Bio Rad Corporation) at the wavelength of $490 \mathrm{~nm}$ to evaluate the cell numbers, which were proportional to the OD values.

\section{Scanning electron microscopy (SEM) of osteoclasts}

After the second, fourth and sixth day, cells on the scaffolds were prepared for SEM imaging to view the respective osteoclasts. The scaffolds were washed twice in PBS and fixed in 2.5\% (v/v) glutaraldehyde in $0.1 \mathrm{M}$ sodium cacodylate buffer for 40 min at $4{ }^{\circ} \mathrm{C}$. The samples were then washed twice in PBS and dehydrated in a graded ethanol series followed by critical point drying with hexamethylsilasane. Following fixation, the scaffolds were sputter coated with gold and viewed on a Leo 1525 Gemini SEM operated at $10 \mathrm{kV}$.

\section{Determination of alkaline phosphatise (ALP) activity}

ALP activity, a marker of osteoblast differentiation and mineralisation, was measured in MC3T3 E1 cultured on scaffolds. All procedures were performed using $6 \times 10^{3}$ MC3T3 E1 cells grown in a 24 well plate for 2,4 and 6 days according to the manufacturer's protocol (Alkaline Phosphatase Fluorometric Assay kit; Abcam, Cambridge, MA).

At the indicated time points, the cells were washed with cold PBS and resuspended in $110 \mu$ l of assay buffer; the cells were rapidly homogenized by pipetting up and down a few times and centrifuged for 3 minutes at $4{ }^{\circ} \mathrm{C}$ at $13000 \mathrm{~g}$ using a cold microcentrifuge to remove any insoluble materials. The supernatant was collected and transferred to a clean tube, then added to each well of a Fluotrac 96-well plate (Corning). Methylumbelliferyl phosphate disodium salt ( $20 \mu \mathrm{l}, 0.5 \mathrm{nM}$ Abcam) substrate was added to each well, followed by incubation for $30 \mathrm{~min}$ at $25^{\circ} \mathrm{C}$; the reaction was stopped by adding $20 \mu \mathrm{l}$ of STOP solution to each well. The fluorescence intensity was measured at $360 \mathrm{~nm}$ excitation and $440 \mathrm{~nm}$ emission using a fluorescence microplate reader (POLARstar Omega, BMG LABTECH).

\section{ELISA for osteocalcin \& osteopontin expression}

The concentrations of osteocalcin (OCN) and osteopontin (OPN) in cell-conditioned culture medium and cells were determined by means of enzyme-linked immunosorbent assay (ELISA) kits (SimpleStep ELISA ${ }^{\mathrm{TM}}$ Kit, Abcam, Cambridge, MA) according to the manufacturer's instructions. All experiments were independently performed in triplicate. Briefly, the cell culture medium was centrifuged at $2000 \mathrm{~g}$ for 10 minutes to remove debris and collect the supernatants and assay. The amount of cells necessary for each total-protein assay was harvested (105 cells); the suspension was centrifuged at $1500 \mathrm{rpm}$ for $10 \mathrm{~min}$ at $4{ }^{\circ} \mathrm{C}$ and the supernatant was aspirated. The pellet was resuspended in cold PBS, transferred to a TPX tube and centrifuged at $1500 \mathrm{rpm}$ for $10 \mathrm{~min}$ at $4{ }^{\circ} \mathrm{C}$. $1 \times$ Cell Extraction Buffer PTR and Protease Inhibitor (Abcam, Cambridge, MA) were added to the ice-cold cell lysis buffer, and the pellet was resuspended and incubated on ice for $10 \mathrm{~min}$. The extraction was stopped after 5 cycles, the supernatant was transferred to a new tube and the samples were centrifuged at $14000 \mathrm{rpm}$ for $15 \mathrm{~min}$ at $4{ }^{\circ} \mathrm{C}$ to remove any remaining insoluble material. An aliquot was taken for quantification. The protein extracts were stored at $-80{ }^{\circ} \mathrm{C}$. Further, $50 \mu \mathrm{l}$ of all samples or standard were added to the appropriate wells; then, $50 \mu \mathrm{l}$ of the antibody cocktail was added 
to each well. The plate was sealed and incubated for 1 hour at room temperature on a plate shaker set to $400 \mathrm{rpm}$. After 1 hour, each well was washed with sufficient $1 \times$ Wash Buffer; then, $100 \mu \mathrm{l}$ of TMB substrate was added to each well, followed by incubation for 10 minutes in the dark on a plate shaker set to $400 \mathrm{rpm}$. Finally, $100 \mu \mathrm{l}$ of stop solution was added to each well. The plate was shaken on a plate shaker for 1 minute for mixing. The results were read on a microplate reader (Ascent) with a test wavelength of $450 \mathrm{~nm}$. Measurements were carried out in three separate wells.

\section{Animals, anesthesia, analgesia, and euthanasia}

All procedures performed on animals were approved by the Animal Ethics Committee at Sichuan University, and the experiments on laboratory animals were performed according to governmental guidelines on animal experiments at Sichuan Province, P. R. China. All experimental animals were New Zealand white rabbits, which were obtained from the experimental animal center in a specific pathogen-free (SPF) colony class and maintained under SPF conditions in a controlled environment. Animals were housed 2 rabbits per cage and were quarantined and acclimatized for 12 weeks. Healthy experimental animals weighing about $2.5 \pm 0.2 \mathrm{~kg}$ were included and grouped randomly; then, the animals were anesthetized with pentobarbital sodium and underwent surgery procedures. Euthanasia of the anesthetized rabbits was accomplished by open thoracic puncture of the heart, and blood collected during this procedure was analysed.

\section{Implantation technique combined subcutaneously (SC) and intramuscularly (IM)}

27 healthy rabbits were randomly grouped into 3 groups and the skin above the implantation sites was shaved with an electrical hair clipper and sterilized with iodine. After the back skin was prepared, a $5 \mathrm{~cm}$ length incision was made on the back of every experimental animal. Then, the unilateral muscle of the spinal column was exposed and an incision was cut on the muscle to make a small pouch, $10 \mathrm{~mm}$ in diameter, which was filled with the prepared scaffolds (CPP group; HA group; SF/CPP group) in the series under study. After the implantation, the wounds were closed. Each group of rabbits was sacrificed after implantation for 4,8 and 12 weeks.

\section{Bone implantation}

Thirty-six healthy rabbits were anesthetized with pentobarbital sodium. For each rabbit, a lengthwise incision was made on the top of the head to expose the skull after a piece of skin was prepped and sterilized with iodine. For bone implantation, a defect of approximately $10 \mathrm{~mm}$ in diameter above the skull was filled with the prepared CPP, HA or SF/CPP scaffold (Fig. 1). In addition, for the control group the skull defects were filled with no scaffold except a blood clot. Each group of rabbits were sacrificed 4, 8 and 12 weeks after implantation. The scaffolds with surrounding tissue were excised, fixed in $10 \%$ neutral buffered formalin, decalcified and embedded in paraffin.

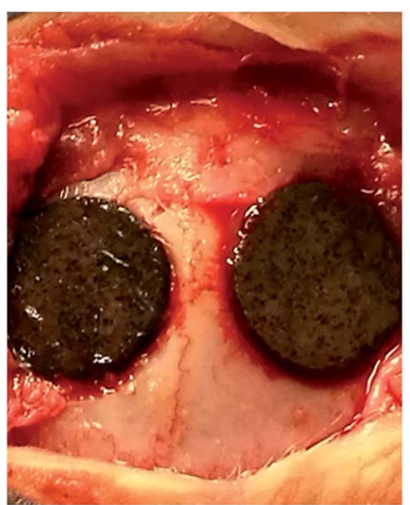

Fig. 1 The defect approximately $10 \mathrm{~mm}$ in diameter above the skull was filled with the prepared scaffold.

\section{Histological evaluations of implant sites}

All implant sites, including the tissues surrounding the implants, were collected and routinely processed for light microscopy. The scaffolds with surrounding tissue were excised, fixed in $10 \%$ neutral buffered formalin, decalcified in $10 \%$ EDTA, decalcified and embedded in paraffin. The embedded tissue blocks were sectioned and stained with hematoxylin and eosin (H\&E) and the histological sections were observed using a light microscope (BX41, Olympus, Japan).

\section{Immunofluorescence staining}

The immunohistochemical procedures employed were introduced in a previous study. Anti-OPN and anti-OCN were purchased from Sigma-Aldrich. The IHC Select® immunoperoxidase Secondary Detection System (Millipore, Germany) was used according to the manufacturer's instructions. Briefly, endogenous peroxidase activity was suspended by incubation in hydrogen peroxide for $10 \mathrm{~min}$. The slides were incubated with anti-OCN or anti-OPN for 10 minutes, steeped in biotinconjugated secondary antibody for $10 \mathrm{~min}$ and then incubated with streptavidin-peroxidase for $10 \mathrm{~min}$ according to the manufacturer's instructions. Finally, colour was developed with a fresh DAB solution for $10 \mathrm{~min}$ and hematoxylin counter stain solution for $1 \mathrm{~min}$. The histological sections were observed using a light microscope (Zeiss, Germany).

\section{Image analysis}

The quantifications of newly formed bone and residual material were performed using image and statistical analysis of the histology samples. After H\&E staining, 10 different locations were randomly selected from each section and observed under the light microscope $(100 \times$ magnification). Using ImageProPlus image analytical software (Media Cybernetics, USA), newly formed bone volume was expressed as the percentage of newly formed bone area in the available pore space (bone area/ pore area $\times 100 \%$ ). All immunofluorescence staining slides were semi-quantitatively evaluated according to the Allred score. The Allred score is obtained by adding the proportion score and intensity score. A total Allred score of 0 to 2 is negative 
and a score of 3 to 8 is positive. Six random fields at $100 \times$ magnification were examined for each tumour and the cells within those fields were counted. ${ }^{\mathbf{1 4}}$

\section{Statistical analysis}

Statistical analysis was performed using SPSS 19.0 software (SPSS, Inc., Chicago, IL). The experimental data obtained in this paper were presented as mean \pm standard deviation (SD). Differences between groups were determined by one-way ANOVA, with a significance level of $p<0.05$.

\section{Results}

\section{MTT assay}

The MTT assay is an efficient method to detect the cytotoxicity of extractions of scaffolds. Fig. 2 presents the cytotoxicity results of CPP and SF/CPP, where HA served as the control. The OD values revealed that the cell proliferation increased with culture time for the three groups. However, it was clear that the growth rate was $\mathrm{SF} / \mathrm{CPP}(1.092 \pm 0.096)>\mathrm{CPP}(0.820 \pm 0.105)>\mathrm{HA}$ $(0.596 \pm 0.063)$ during the entire culture period, indicating that $\mathrm{SF} / \mathrm{CPP}$ was nontoxic. There was a statistical difference between each group at co-culture on the 6th day. The one-way ANOVA quantitative analysis of the MTT assay revealed that the growth rate of the cells co-cultured with SF/CPP scaffolds was significantly higher than those of the other groups $(p<0.05)$. In addition, SF/CPP exhibited better biocompatibility in vitro than CPP and HA.

\section{ALP activity}

MC3T3 E1 cells cultured on the scaffolds showed increased total ALP activity with Bombyx mori silk doping. There were no significant differences in total ALP per disc after 2 days in culture; however, after 4 weeks, cells on the SF/CPP scaffolds had higher total ALP activity than those on the CPP or HA scaffolds, without a statistically significant difference. After 6 days in culture, cells on the SF/CPP scaffolds had significantly higher total ALP activity than those on the CPP or HA scaffolds. This trend held when the ALP activity was normalised per cell number. There was a statistical difference between each group at co-culture 6th day, and one-way ANOVA quantitative analysis

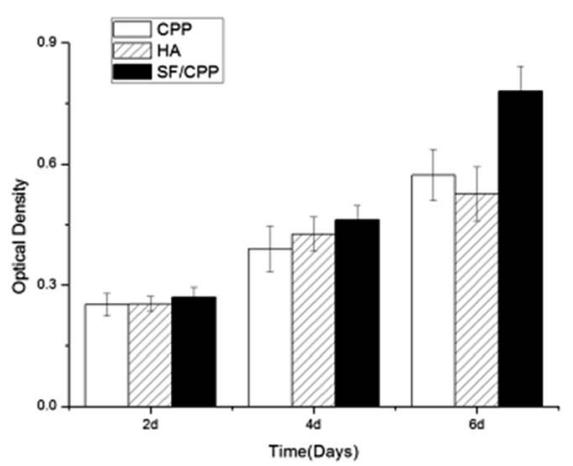

Fig. 2 MTT assay for proliferation of MC3T3 E1 cultured in the extractions of CPP, HA and SF/CPP for different days.

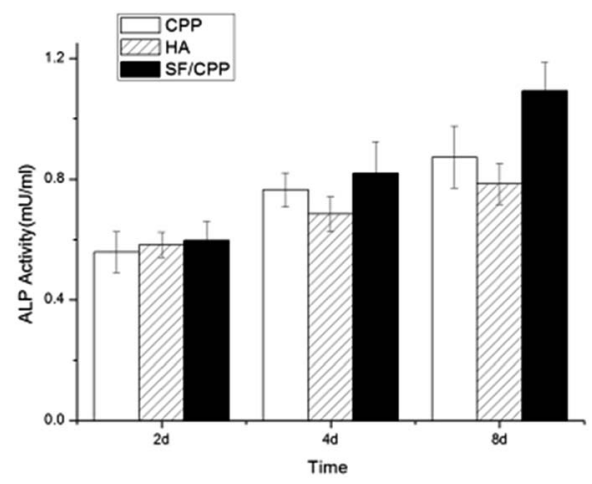

Fig. 3 The effects of the scaffolds on alkaline phosphatase (ALP) activity in mice osteoblast MC3T3 E1 cultures for different days.

of the ALP activity revealed that the cells co-cultured with SF/ CPP scaffolds were significantly higher than those of other groups $(p<0.05)$ (Fig. 3).

\section{SEM of osteoclasts}

MC3T3 E1 monocytes cultured on SF/CPP differentiated to large cells with apico-basal polarisation; this morphology is typical of resorbing osteoclasts. After 6 days in culture, the number of cells attached on the surfaces of the SF/CPP scaffolds was significantly higher than those on the CPP or HA scaffolds. Many undifferentiated monocytes were also visible (Fig. 4).

\section{OPN and OCN protein expression levels in vitro}

The protein expressions of OPN and OCN of MC3T3 E1 cells on the CPP, HA and SF/CPP scaffolds increased over the six-day culture period for all scaffolds. Cell immunohistochemical studies showed that the OPN positive cells had higher expression than those on the CPP or HA scaffolds after co-culture for 6 days; the same trend was observed in the OCN positive cells (Fig. 5). We used ELISA to quantitatively analyse the concentrations of OPN and OCN proteins in the supernatant (Fig. 6). After 2 days in culture, there were no significant differences between the number of cells on any scaffolds. However, after 4 days in culture,

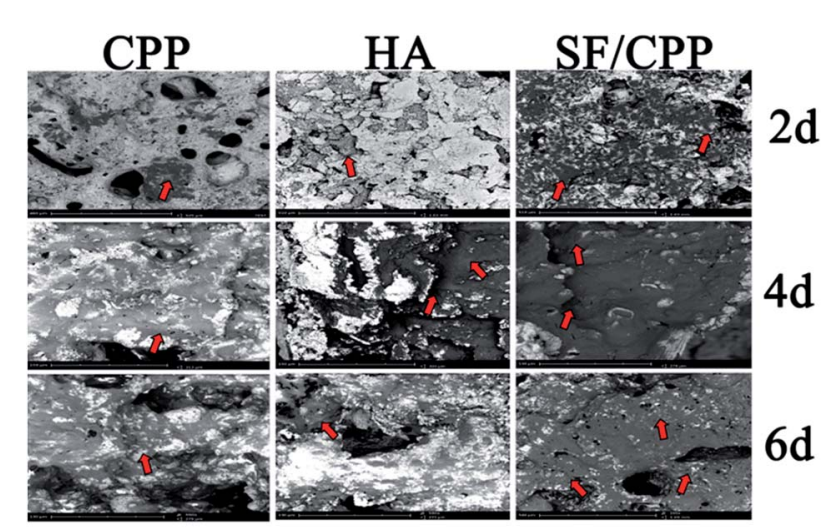

Fig. 4 Scanning electron micrographs of CPP, HA and SF/CPP for different days (the series of red arrows indicates dehydrated MC3T3 E1 cells). 


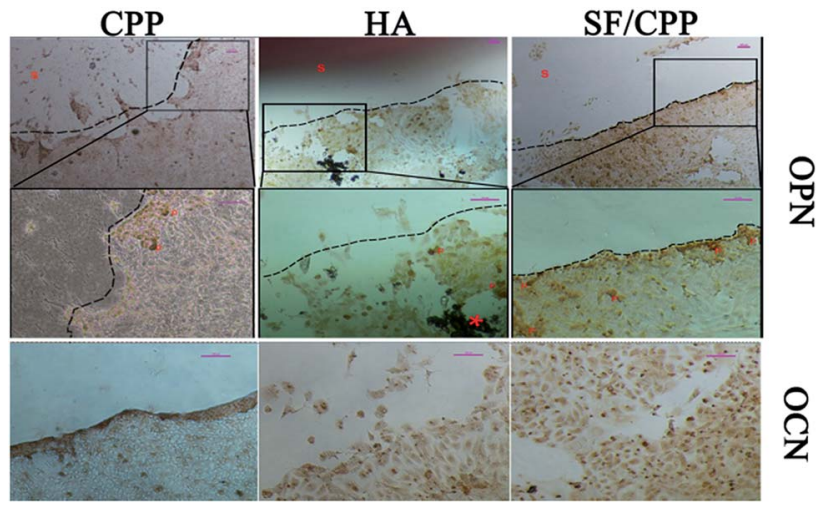

Fig. 5 Optical micrographs of OPN and OCN expression of CPP, HA and SF/CPP scaffolds co-cultured with MC3T3 E1 after 6 days (s: scaffolds; p: positive cells; *: scattered scaffolds).
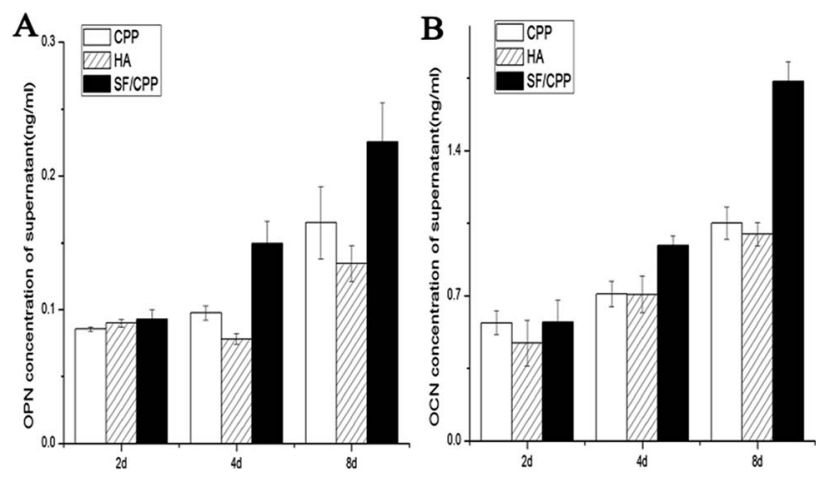

Fig. 6 OPN (A) and OCN (B) expression in the supernatants detected by ELISA in each group of CPP, HA and SF/CPP scaffolds co-cultured with MC3T3 E1.

there were significantly more cells on the SF/CPP scaffolds than on the CPP and HA scaffolds, which comparatively increased after 6 days in culture. The protein expressions of OPN and OCN in the supernatant increased gradually in all tested groups, indicating that the cells co-cultured with SF/CPP had the highest OPN and OCN concentrations in the supernatant among the groups at the 6 th day $(p<0.05)$.

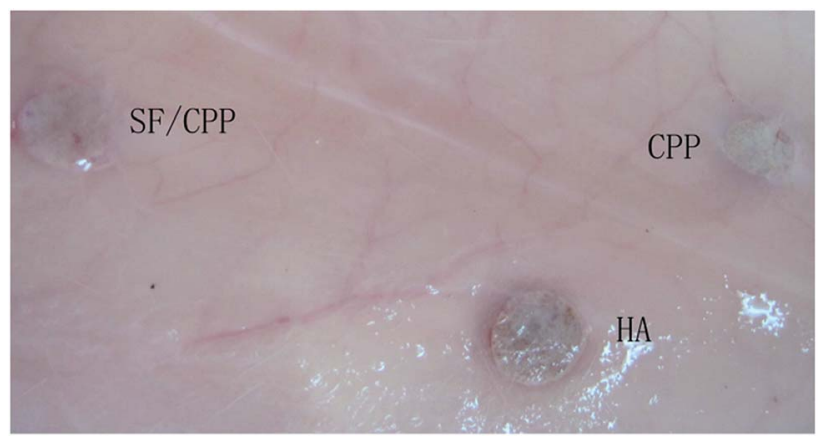

Fig. 7 Clinical specimen photographs of CPP, HA and SF/CPP scaffolds after subcutaneous implantation after 12 weeks.

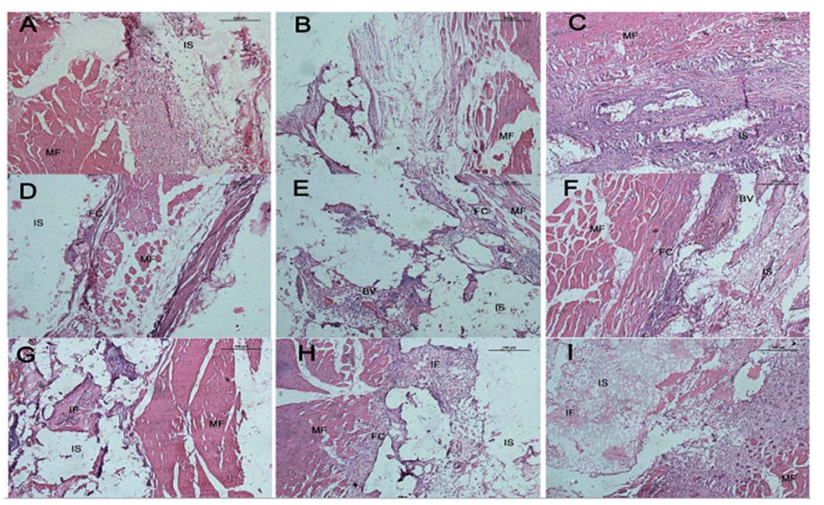

Fig. 8 H\&E histological sections of tissues surrounding implant 4 (AC), $8(D-F)$, and $12(G-I)$ weeks after intramuscular implantation in rabbits $(\times 100)$ (CPP: A, D and G; HA: B, E and H; SF/CPP: C, F and I; MF: muscle fiber; BV: newly formed blood vessels; FC: fibrous capsule; IS: implanted scaffold; IF: inner fiber in the scaffold cavity).

\section{Intramuscular and subcutaneous implantation}

All animals survived until the scheduled date of sacrifice, with no evidence of acute inflammation and toxicity in any group. Fig. 7 demonstrates the clinical specimens of the CPP, HA and SF/CPP scaffolds in the subcutaneous region after the 12 week analysis period. Clinical evidence of induced changes in physical appearance was not observed. Complications and adverse events associated with implantation, such as wound infection, incision dehiscence, and hydrocephalus, were absent throughout the study.

In the muscle implantation experiment, all implant sites exhibited scaffolds at the time of harvest. Fig. 8 shows the histological evaluation of the scaffolds and surrounding tissues in muscle pouches after implantation for 12 weeks. The SF/CPP scaffold-implanted tissues exhibited the typical structure of large fibrous tissues filled with vessels surrounded by a combination of implanted SF/CPP scaffolds (at 4 weeks) and muscle without the presence of new bone or a marrow cavity during the 12 week analysis period. The complete material structure could be clearly observed after 8 weeks; furthermore, each of these areas could be separated between the materials and tissues in SF/CPP scaffold-implanted tissues after 12 weeks. The fibrous tissues were closely linked with the muscle fibers under the microscope. In the HA and CPP groups, the fibrous capsule interface between the materials and tissues was still clearly visible after implantation for 12 weeks. The CPP scaffoldimplanted group produced much more variable results. In the CPP group, inner acellular amorphous material structures were observed after 8 and 12 postoperative weeks; also, there were some fibrous tissues with large pores. In some cases, small amounts of new fibrous tissues were formed in the HA scaffold. However, a significantly large number of giant cells were formed around the SF/CPP scaffolds than the HA and CPP scaffolds from week 4 . All the evidence indicated that the scaffolds containing SF/CPP were nontoxic.

Fig. 9 shows representative images of OPN and OCN immunohistochemical stains of the CPP, HA and SF/CPP scaffolds after 


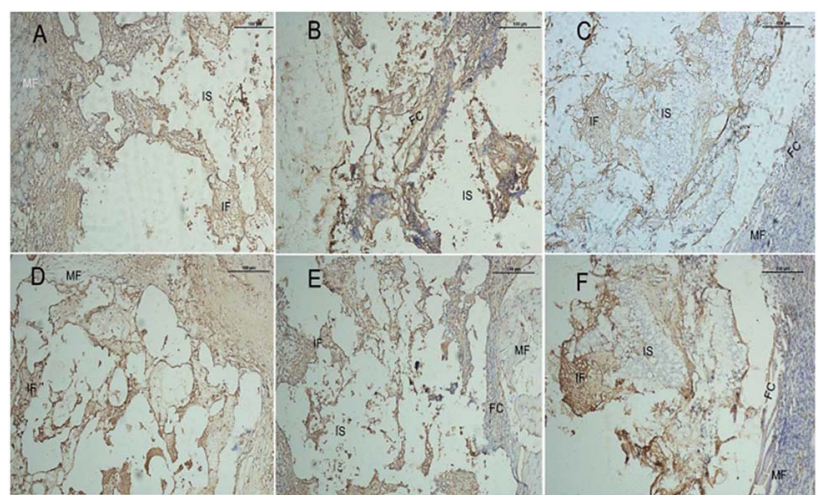

Fig. 9 Optical micrographs of OPN (A-C) and OCN (D-F) stained sections of scaffolds 12 weeks after intramuscular implantation in rabbits $(\times 100)$ (CPP: $A$ and $D$; HA: $B$ and $E$; SF/SCPP: $C$ and F; MF: muscle fiber; BV: newly formed blood vessels; FC: fibrous capsule; IS: implanted scaffold; IF: inner fiber in the scaffold cavity).

12 week IM implantation, on which the positive immunohistochemistry staining for OPN and OCN was observed in newly regenerated tissue in both the CPP group and HA group with more intensive positive staining in the HA group than the CPP group. In contrast, the complete structure of the scaffold was still clear in the SF/CPP group at week 12, and the immunohistochemistry staining for OPN and OCN in newly regenerated tissue in the SF/CPP group presented mild positives. A few fibres were adhered to the scaffold, providing it more tenacity. The implantation of any biomaterial will cause an inflammatory response, known as foreign body reaction (FBR). However, the SF/CPP demonstrated stability to degradation and maintained a better scaffold structure under the microscope. Therefore, we speculate that $\mathrm{SF} / \mathrm{CPP}$ is a better option for cells and maintains a superior three-dimensional structure compared with CPP or HA.

Four weeks after SC implantation, the SF/CPP implant was located in a clearly defined cavity, which is characterized by a maximal presence of complete structure material (Fig. 10). The implant was enveloped by a fibrous capsule (FC) consisting mainly of neutrophils, proliferating fibroblasts, scant collagen

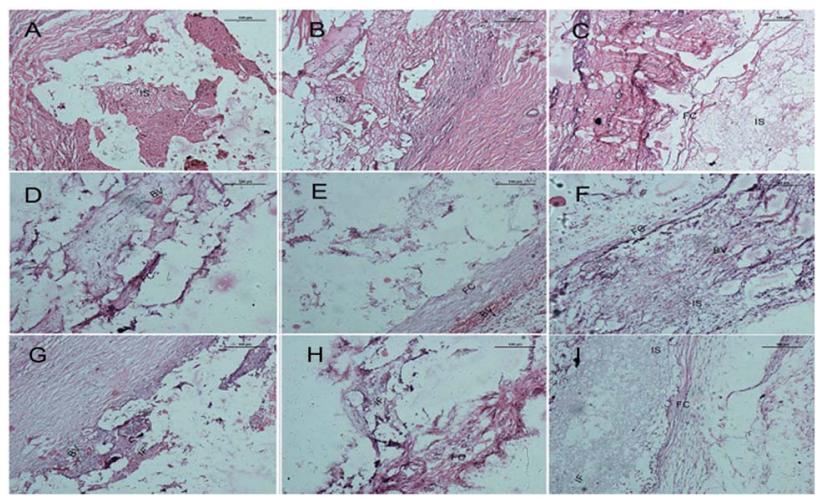

Fig. 10 H\&E histological sections of tissues surrounding implant 4 (AC), $8(D-F)$, and $12(G-I)$ weeks after subcutaneous implantation in rabbits $(\times 100)$ (CPP: A, D and G; HA: B, E and H; SF/SCPP: C, F and I; BV: newly formed blood vessels; FC: fibrous capsule; IS: implanted scaffold; IF: inner fiber in the scaffold cavity). deposition and activated macrophages (Fig. 10). No necrosis was observed and there was no presence of acute inflammatory cells at the outer surface of the FC. After 4 weeks, relatively thick FC was observed to surround the CPP and HA implants. The tissue response was dominated by a proliferating fibroblastic reaction, which is associated with collagen deposition and also included a minimal to moderate presence of acellular amorphous material in the scaffold cavity. The scaffold degradation time was slower than that of SF/CPP. On week 8, histological examination revealed a substantial decrease in the intensity of the inflammatory response. The CPP and HA scaffolds were surrounded by FC characterized predominantly by the proliferating fibroblastic reaction, which is associated with prominent collagen deposition and included a minimal presence of acellular amorphous material in the scaffold cavity (Fig. 10). Moreover, the SF/CPP implant was located in a clearly defined cavity, which is characterized by the clear presence of a complete scaffold structure. Inner fibers in the scaffold cavity were observed in the center of the scaffold (Fig. 10). After 12 weeks of SC implantation, the adjacent tissue response was similar to that observed at week 8; the CPP and HA scaffolds were degradable and non-toxic resins as well as the complete structure could not be observed (Fig. 10). After 12 weeks of implantation, the H\&E stain results indicated that the most evident fibrous tissues in the scaffold cavity were observed in the SF/CPP group compared with the CPP and HA groups (Fig. 10). Meanwhile, complete scaffold structures were observed in the SF/ CPP scaffolds after implantation for 12 weeks. All the evidence indicated that the scaffolds containing SF/CPP were nontoxic.

Fig. 11 shows representative images of the OPN and OCN immunohistochemical stains of the CPP, HA and SF/CPP scaffolds 12 weeks after SC implantation. Both fiber formation and VEGF-evidence of angiogenesis in vivo in the scaffolds and in the tissues surrounding the scaffolds could be observed. After 12 weeks of implantation, the positive immunohistochemistry staining for OPN and OCN were found in newly regenerated tissue in both the CPP group and HA group with more intensive positive staining in the HA group than in the CPP group. In contrast, the complete structure of the scaffold was still clear in

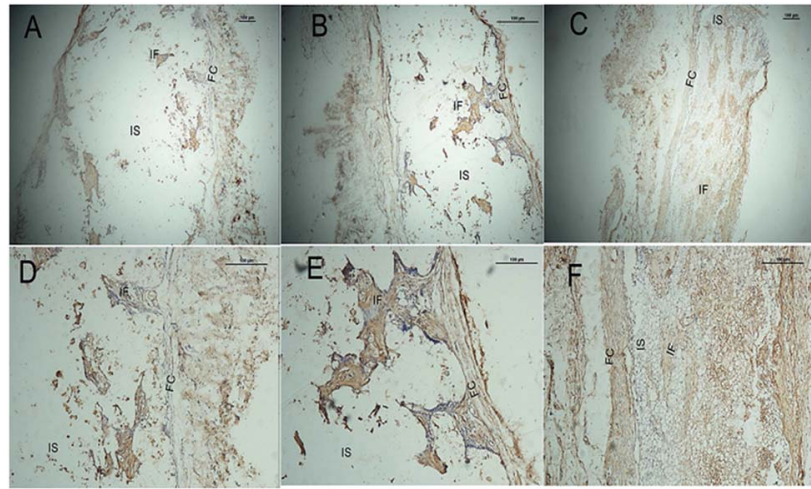

Fig. 11 Optical micrographs of OPN $(A-C)(\times 40)$ and OCN $(D-F)$ $(\times 100)$ stained sections of the scaffolds 12 weeks after intramuscular implantation in rabbits (CPP: A and D; HA: B and E; SF/CPP: C and F; MF: muscle fiber; BV: newly formed blood vessels; FC: fibrous capsule; IS: implanted scaffold; IF: inner fiber in the scaffold cavity). 
the SF/CPP group at 12 weeks; the immunohistochemistry staining for OPN and OCN in the newly regenerated tissue in the SF/CPP group presented a mild positive. After 12 weeks, histological examination revealed that the CPP and HA scaffolds were surrounded by FC characterized predominantly by the proliferating fibroblastic reaction, which is associated with prominent collagen deposition and included a minimal presence of acellular amorphous material in the scaffold cavity (Fig. 5). Moreover, the SF/CPP implant was located in a clearly defined cavity characterized by the clear presence of a complete scaffold structure. Due to the degradation and inner fiber formation of SF/CPP, the materials were more stable. Therefore, compared with the implanted CPP and HA scaffolds, higher expressions of the angiogenic growth factors OPN and OCN were present in the HA scaffold.

\section{Bone implantation}

After 4 weeks of implantation, a new bone regenerated and penetrated through the inter-connective pores to the centers of

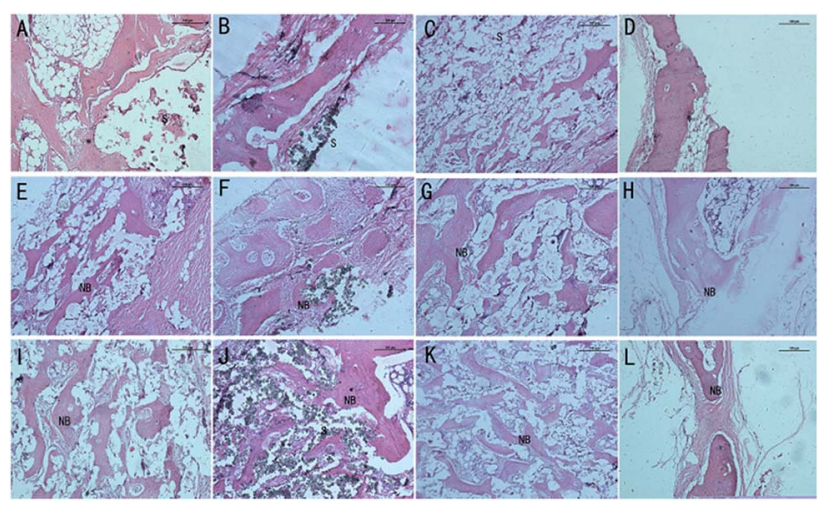

Fig. 12 Histological sections of the CPP, HA and SF/CPP scaffolds and the control group $4(A-D), 8(E-H)$ and $12(I-L)$ weeks after bone implantation in rabbits (CPP: A, E and I; HA: B, F and J; SF/CPP: C, G and $K$; control: $D, H$ and L). NB: newly formed bone; s: scaffolds.

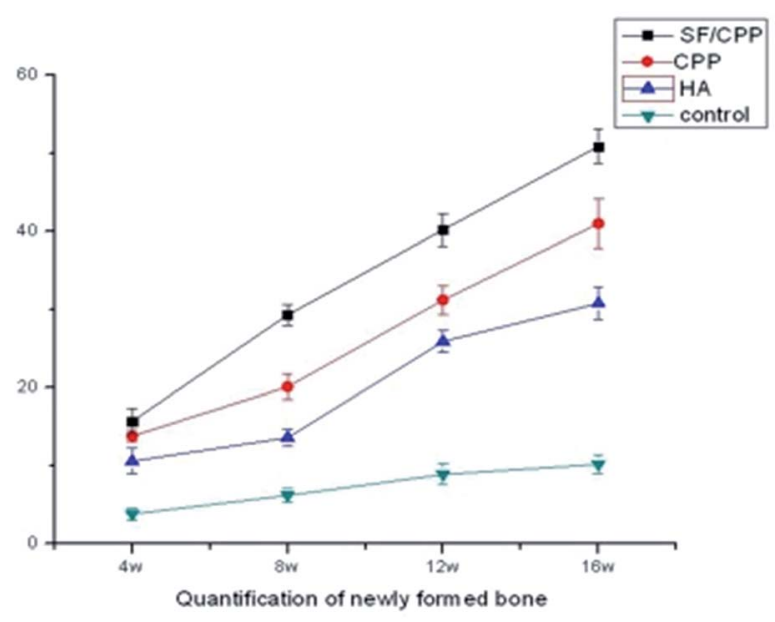

Fig. 13 Quantification of newly formed bone by statistical analysis of the histological sections. Error bars represent mean \pm SD for $n=3$. the scaffolds. In the case of the SF/CPP group, the interface between the material and the host bone was hardly detectable and formed a close union without any gap. New large areas of bone in the center of the scaffolds, with trabecular structures encasing the scaffolds, were consistently seen. In the case of the CPP group, the host bone formed a close union, while the new bone regenerated and penetrated through the inter-connective pores to the margins of the scaffolds. Moreover, in the centers of the materials, no new bone or degradation of the CPP and HA scaffolds was observed (Fig. 12). Fig. 13 shows that the newly formed bone grew larger in each group during the implantation period. These results demonstrated that the SF/CPP scaffolds presented more efficient bone formation than the CPP and HA scaffolds at the 12 week analysis period.

Clearly, before 8 weeks post implantation, the amounts of newly formed bone in the SF/CPP group increased dramatically, much more than those of the CPP scaffolds. OPN and OCN positive cells (osteoblasts) were observed in the centers of the scaffolds of SF/CPP and the newly formed bone structures

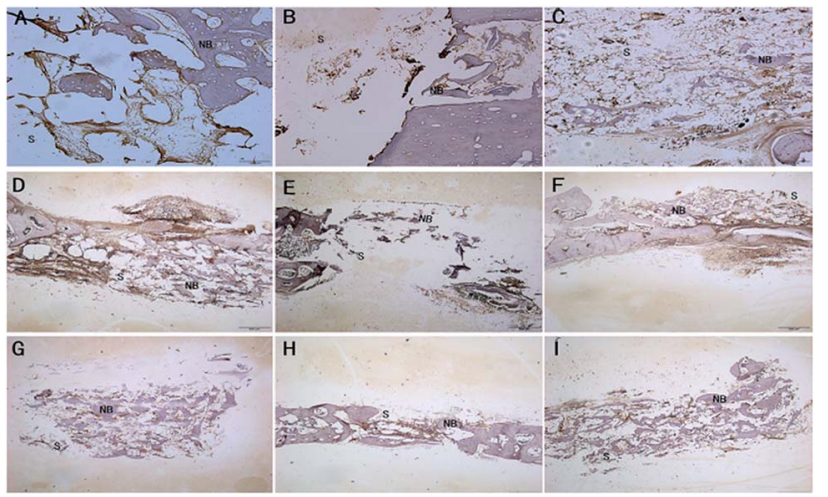

Fig. 14 Optical micrographs of OPN stained sections of the CPP, HA and SF/CPP scaffolds $4(A-C), 8(D-F)$ and $12(G-I)$ weeks after bone implantation in rabbits (CPP: A $(\times 100), D(\times 100)$ and $G(\times 40)$; HA: B ( $\times 100), E(\times 100)$ and $H(\times 40)$; SF/CPP: $C(\times 100), F(\times 100)$ and I $(\times 40)$; NB: newly formed bone; s: scaffolds).

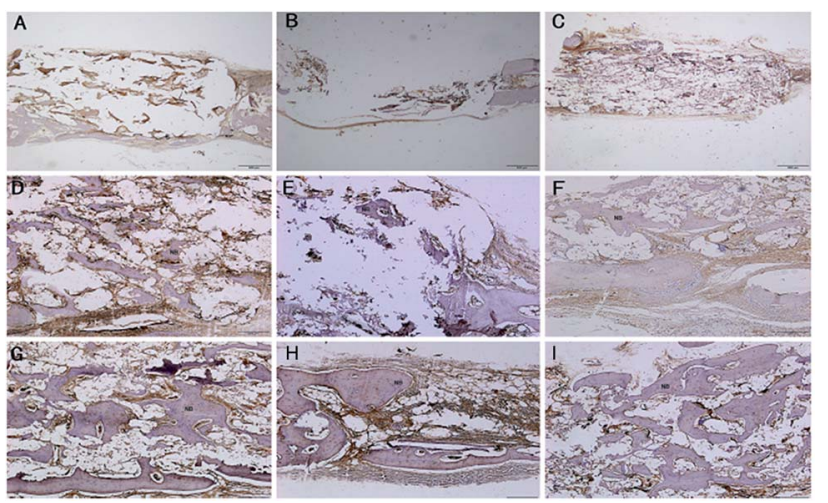

Fig. 15 Optical micrographs of OCN stained sections of CPP, HA and SF/CPP scaffolds after $4(A-C), 8(D-F)$ and $12(G-I)$ weeks of bone implantation in rabbits (CPP: A $(\times 40), D(\times 40), G(\times 100)$; HA: B $(\times 40), E$ $(\times 40)$ and $H(\times 100)$; SF/CPP: $C(\times 40), F(\times 40)$ and I $(\times 100)$; NB: newly formed bone; s: scaffolds). 
around the positive cells in the SF/CPP group. While it was difficult to find positive cells in the centres of the CPP and HA scaffolds, more fibers were observed in the porous spaces of the CPP scaffolds. At 8 weeks post-implantation, the amount of newly formed bone in the SF/CPP group was higher than those in the CPP and HA scaffolds. Following this period, however, the rate of new bone formation in the SCPP scaffolds slowed, while in the CPP scaffolds the rate of new bone formation gradually increased. These results indicated that the SF/CPP scaffolds present more efficient bone formation than the CPP and HA scaffolds in the long term (12 week analysis period) (Fig. 14 and 15). The levels of OPN and OPN expression were assessed by Allred score. OPN and OCN expression were associated with newly formed bone with mean Allred scores of 6.78, 4.24 and 7.32 in the CPP, HA and SF/CPP groups, respectively (all positive).

\section{Discussions}

Apatite bioceramics have been widely researched as repairing materials in bone tissue engineering. In recent applications of bioceramics in bone tissue engineering, studies have shown the use of porous materials with bone-like structures and apatite surfaces to activate and regulate the cascade expression of osteogenic genes; these porous materials guide the cells to differentiate along the osteogenic lineage in coordination with the biological environment. ${ }^{13}$ The newly formed bone induced by the scaffold was identified as the extracellular matrix production of differentiated osteoblasts adhering on its surface. ${ }^{15}$ The ideal bone scaffold material should have sufficient graft strength with a bone-like structure as well as an apatite surface to activate and regulate the cascade expression of osteogenic genes at a preferred rate to eventually be replaced by newly formed natural bone. ${ }^{3}$ Over the past decades, numerous investigations have been conducted to find materials that could be used as replacement material grafts with low deterioration rates and strong durability. In this context, various synthetic materials that were not previously used for biomedical purposes were thus evaluated. In addition, biological scaffolds or hybrid composites were evaluated for use in tissue engineering. For the latter application, other synthetic polymers, such as polyglycolic resorbable prostheses, produced poor results in clinical tests with early inflammatory reactions, short lifespan, and limited effectiveness as a result of their breakdown products. In this context, we have investigated a new hybrid material composed of CPP crosslinked with silk proteins.

Although their fragility and low self-degradation limits further applications, CPP scaffolds could be prepared for use in bone tissue-engineering. ${ }^{16}$ In a previous study, CPP-type bioceramics presented higher compressive strength and enhanced degradation after silk fibroin was doped into the CPP structure. In vitro results indicated that the silk fibroin increased the compressive strength as well as the degradation behavior and biocompatibility of the structure. ${ }^{12} \mathrm{SF} / \mathrm{CPP}$ was designed to serve as a bone scaffold, guiding the migration, adhesion, and proliferation of osteoblasts; in addition, SF/CPP showed significantly higher degradation than CPP scaffolds at $30^{\text {th }}$ day. ${ }^{12}$ The purpose of this study was to develop degradable bioceramics using silk fibroin
(SF) applied in CPP scaffolds. We aimed to investigate the biocompatibility and osteogenesis in vitro and in vivo and explore the osteogenesis effects of OPN and OCN expression, the relationship between OPN and OCN expression and the rate of newly formed bone with the primarily examined mechanisms.

The present study indicated an increase of ALP activity in osteoblasts, enhancing the rate of new bone formation in bone remodelling. ${ }^{17,18}$ The previous studies were intended to detect ALP activity in serum, bones, and bone cells and the number of ALP-positive colony-forming units in bone marrow stromal cell cultures; the results indicated that ALP activity is a product of osteoblasts and has been shown to correlate with rates of bone formation. ${ }^{17}$ In this study, significant differences were observed between the different scaffold co-cultures with pre-osteoblasts. With hydrophilic silk fibroin doped into the CPP scaffolds, the ALP activity increased compared to the CPP and HA groups. This suggests that SF doped in the CPP scaffolds increased the alkaline phosphatase activity of osteoblasts in vitro.

$\mathrm{SF}$ as an individual material has been used extensively in bone tissue-engineering approaches and has been shown to facilitate bone formation in vitro and in vivo. ${ }^{19} \mathrm{SF}$ was employed to regulate the mineralization of HA nanocrystals. In addition, SF has been used as a dopant to enhance the mechanical properties of SF/HA composite scaffolds. ${ }^{20,21}$ However, the rate at which the HA scaffold was replaced by the newly formed natural bone was undesirably slow during the bone repair procedure because of its low degradability.

According to a previous study, the physical state of collagen has a dramatic outcome on the degradation rate and provokes a totally different foreign body reaction (FBR). The FBR has three phases: onset, progression and resolution. ${ }^{22,23}$ Although CPP and HA have been recognised as non-toxic and biocompatible scaffolds, our current data showed that they both present an unstable feature in the FBR due to the following factors: slow onset (as reflected by slow degradation and capsulation) and advanced progression (as reflected by fast degradation). In contrast, SF/CPP demonstrated stability to degradation and maintained a better scaffold structure under the microscope; therefore, we speculate that SF/CPP is a better homing scaffold in comparison with CPP or HA for cells to maintain three-dimensional structures.

Previous studies have also demonstrated that OPN is highly enriched at regions of the bone surface where osteoclasts are anchored and localizes at the corresponding area of the osteoclast plasma membrane, ${ }^{12}$ supporting that OPN plays a pivotal role in bone formation. ${ }^{24,25}$ The OCN gene leads to truncated proteins and development of Keutel syndrome, characterized by abnormal cartilage calcification, peripheral pulmonary stenosis, and midfacial hypoplasia. ${ }^{26}$ On the other hand, the sustained presence of OCN has been shown to enhance osteoblast differentiation and the corresponding mineral contents. ${ }^{27}$ A similar study also indicated that silk fibroin/HA scaffolds promote the osteoblast-related gene markers (OPN and OCN) and ALP activities and show great potential as osteogenesis promoting scaffolds. ${ }^{28}$ Therefore, the expression of OPN and OCN has become an important indication which measures the ability of scaffolds to induce bone formation in vivo. In this study, different expression patterns of OPN and OCN were observed in the three sample groups. The expressions 
of OPN and OCN within the SF/CPP scaffold were significantly higher and more widely and evenly distributed than those of the CPP and HA scaffolds during the post-operation periods in vivo and during co-culture with pre-osteoblasts after 6 days in vitro. In our results, the SF/CPP scaffold enhanced the differentiation of pre-osteoblasts in vitro and increased the newly formed bone in vivo with increased expression of OPN and OCN. This indicates that the positive effects of SF/CPP scaffolds for osteoblasts are associated with OPN and OCN. In addition, the SF/CPP group exhibited better effects than the CPP and HA groups, in positive correlation with the expression of OPN and OCN in vitro and in vivo, suggesting that $\mathrm{SF}$ could activate and regulate the cascade expression of the osteogenic genes of OPN and OCN and guide the cells to differentiate between them.

\section{Conclusions}

The current study introduces novel CPP-based bioceramics doped with SF that were prepared for bone tissue-engineering. In a previous study, in vitro results indicated that the silk fibroin increased the compressive strength and the degradation behavior and biocompatibility of the bioceramics. It was found that OPN and OCN expression were effectively enhanced in vitro when cocultured with pre-osteoblasts. In addition, the positive effects of increasing OPN and OCN expression enhanced the newly formed bone volume; this was further evaluated by in situ application using the bone defect model in vivo. The in vivo results indicated that SF/CPP showed better tissue biocompatibility, biodegradability, osteoinductivity and osteoconductivity. The SF/CPP scaffold showed comparably good biocompatibility and osteogenicity than the hybrid scaffolds; this indicates that the SF/CPP scaffold fulfils the basic requirements of a bone tissue engineering scaffold. Collectively, our study shows that SF/CPP is an effective method to promote newly formed bone volume and could be a good candidate for bone implantation, thus providing a feasible therapeutic approach for treating patients with bone defects.

\section{Conflicts of interest}

There are no conflicts to declare.

\section{Acknowledgements}

The authors would like to thank the National Natural Science Foundation of China (No. 51641305), the youth fund of Sichuan University, the youth fund of the West China School of Stomatology, and the Rising Stars Leadership grant of James Cook University (JCURSP12YQ).

\section{Notes and references}

1 Y. Zhang, X. Cui, S. Zhao, H. Wang, M. N. Rahaman, Z. Liu, W. Huang and C. Zhang, ACS Appl. Mater. Interfaces, 2015, 7(4), 2393-2403.

2 R. Pilliar, M. Filiaggi, J. Wells, M. Grynpas and R. Kandel, Biomaterials, 2001, 22(9), 963-972.
3 M. Grynpas, R. Pilliar, R. Kandel, R. Renlund, M. Filiaggi and M. Dumitriu, Biomaterials, 2002, 23(9), 2063-2070.

4 E. K. Park, Y. E. Lee, J.-Y. Choi, S.-H. Oh, H.-I. Shin, K.-H. Kim, S.-Y. Kim and S. Kim, Biomaterials, 2004, 25(17), 3403-3411.

5 A. Dias, M. Lopes, I. Gibson and J. Santos, J. Non-Cryst. Solids, 2003, 330(1), 81-89.

6 K. Qiu, C. Wan, C. Zhao, X. Chen, C. Tang and Y. Chen, J. Mater. Sci., 2006, 41(8), 2429-2434.

7 H. Xie, Q. Wang, Q. Ye, C. Wan and L. Li, J. Mater. Sci.: Mater. Med., 2012, 23(4), 1033-1044.

8 S. D. Waldman, M. D. Grynpas, R. M. Pilliar and R. A. Kandel, J. Biomed. Mater. Res., 2002, 62(3), 323-330.

9 X. Wang, J. A. Kluge, G. G. Leisk and D. L. Kaplan, Biomaterials, 2008, 29(8), 1054-1064.

10 M. Cattani-Lorente, R. Rizzoli and P. Ammann, Acta Biomater., 2013, 9(6), 7005-7013.

11 G. H. Altman, R. L. Horan, H. H. Lu, J. Moreau, I. Martin, J. C. Richmond and D. L. Kaplan, Biomaterials, 2002, 23(20), 4131-4141.

12 H. Xie, Z. Gu, C. Li, C. Franco, J. Wang, L. Li, N. Meredith, Q. Ye and C. Wan, Ceram. Int., 2016, 42(2), 2386-2392.

13 H. Zreiqat, Y. Ramaswamy, C. Wu, A. Paschalidis, Z. Lu, B. James, O. Birke, M. McDonald, D. Little and C. R. Dunstan, Biomaterials, 2010, 31(12), 3175-3184.

14 W. Li, F. Chen, F. Zhang, W. Ding, Q. Ye, J. Shi and B. Fu, PLoS One, 2013, 8(4), e60962.

15 H. Xie, J. Wang, C. Li, Z. Gu, Q. Chen and L. Li, Ceram. Int., 2013, 39(8), 8945-8954.

16 X. Wang, Z. Gu, B. Jiang, L. Li and X. Yu, Biomater. Sci., 2016, 4(4), 678-688.

17 D. Gopi, S. Ramya, D. Rajeswari and L. Kavitha, Colloids Surf., B, 2013, 107(4), 130-136.

18 Z. Gu, B. Huang, Y. Li, M. Tian, L. Li and X. Yu, Mater. Sci. Eng., C, 2016, 61, 526-533.

19 P. Aramwit, T. Siritientong, S. Kanokpanont and T. Srichana, Int. J. Biol. Macromol., 2010, 47(5), 668-675.

20 S. Bhumiratana, W. L. Grayson, A. Castaneda, D. N. Rockwood, E. S. Gil, D. L. Kaplan and G. Vunjak-Novakovic, Biomaterials, 2011, 32(11), 2812-2820.

21 H. Zreiqat, Y. Ramaswamy, C. Wu, A. Paschalidis, Z. F. Lu, B. James, O. Birke, M. Mcdonald, D. Little and C. R. Dunstan, Biomaterials, 2010, 31(12), 3175-3184.

22 Q. Ye, M. C. Harmsen, Y. Ren and R. A. Bank, Biomaterials, 2011, 32(5), 1339-1350.

23 Q. Ye, M. J. van Amerongen, J. A. Sandham, R. A. Bank, M. J. van Luyn and M. C. Harmsen, J. Tissue Eng. Regener. Med., 2011, 5(4), 264.

24 J. Kanis, Osteoporosis Int., 2014, 22(8), 2347-2355.

25 M. D. Patrick Ammann, I. Badoud, S. Barraud, R. Dayer and R. Rizzoli, J. Bone Miner. Res., 2007, 22(9), 1419-1425.

26 M. B. Camargo, T. Vilaça, L. F. Hayashi, O. G. Rocha and M. Lazaretti-Castro, J. Bone Miner. Metab., 2014, 33(3), 319-328.

27 S. G. Dahl, P. Allain, P. J. Marie, Y. Mauras, G. Boivin, P. Ammann, Y. Tsouderos, P. D. Delmas and C. Christiansen, Bone, 2001, 28(4), 446-453.

28 X. Ding, C. Wu, H. Tong, L. Wang, Y. Huang, H. Kang, et al., RSC Adv., 2016, 6(23), 19463-19474. 